This is an electronic reprint of the original article.

This reprint may differ from the original in pagination and typographic detail.

Author(s): Elder, K. R. \& Rossi, G. \& Kanerva, P. \& Sanches, F. \& Ying, S-C. \& Granato, E. \& Achim, C. V. \& Ala-Nissilä, Tapio

Title: $\quad$ Modeling self-organization of thin strained metallic overlayers from atomic to micron scales

Year: $\quad 2013$

Version: $\quad$ Final published version

Please cite the original version:

Elder, K. R. \& Rossi, G. \& Kanerva, P. \& Sanches, F. \& Ying, S-C. \& Granato, E. \& Achim, C. V. \& Ala-Nissilä, Tapio. 2013. Modeling self-organization of thin strained metallic overlayers from atomic to micron scales. Physical Review B. Volume 88, Issue 7. P. 075423/1-10. ISSN 1098-0121 (printed). DOI: 10.1103/physrevb.88.075423.

Rights:

(C) 2013 American Physical Society (APS). http://www.aps.org

All material supplied via Aaltodoc is protected by copyright and other intellectual property rights, and duplication or sale of all or part of any of the repository collections is not permitted, except that material may be duplicated by you for your research use or educational purposes in electronic or print form. You must obtain permission for any other use. Electronic or print copies may not be offered, whether for sale or otherwise to anyone who is not an authorised user. 


\title{
Modeling self-organization of thin strained metallic overlayers from atomic to micron scales
}

\author{
K. R. Elder, ${ }^{1}$ G. Rossi, ${ }^{2}$ P. Kanerva, ${ }^{2}$ F. Sanches, ${ }^{1}$ S-C. Ying, ${ }^{3}$ E. Granato,,${ }^{3,4}$ C. V. Achim, ${ }^{2}$ and T. Ala-Nissila ${ }^{2,3}$ \\ ${ }^{1}$ Department of Physics, Oakland University, Rochester, Michigan 48309-4487, USA \\ ${ }^{2}$ Department of Applied Physics and COMP Centre of Excellence, Aalto University School of Science, \\ P.O. Box 11000, FI-00076 AALTO, Finland \\ ${ }^{3}$ Department of Physics, Brown University, P.O. Box 1843, Providence, Rhode Island 02912-1843, USA \\ ${ }^{4}$ Laboratório Associado de Sensores e Materiais, Instituto Nacional de Pesquisas Espaciais, 12227-010 São José dos Campos, SP, Brazil
}

(Received 7 May 2013; published 16 August 2013)

\begin{abstract}
A computational study of the self-organization of heteroepitaxial ultrathin metal films is presented. By means of a continuum complex field model, the relationship of the equilibrium surface patterns of the film to the adsorbate-substrate adhesion energy, as well as to the mismatch between the adsorbate and the substrate bulk lattice parameters, are obtained in both the tensile and the compressive regimes. Our approach captures pattern periodicities over large length scales, up to several hundreds of $\mathrm{nm}$, retaining atomistic resolution. Thus, the results can be directly compared with experimental data, in particular for systems such as $\mathrm{Cu} / \mathrm{Ru}(0001)$ and $\mathrm{Ag} / \mathrm{Cu}(111)$. Three nontrivial, stable superstructures for the overlayer, namely, stripe, honeycomb, and triangular, are identified that closely resemble those observed experimentally. Simulations in nonequilibrium conditions are performed as well to identify metastable structural configurations and the dynamics of ordering of the overlayer.
\end{abstract}

DOI: 10.1103/PhysRevB.88.075423

PACS number(s): 68.55.-a, 81.10.Aj, 68.35.bd

\section{INTRODUCTION}

Ultrathin metal films have been the object of intense investigation for many years as the interaction of the substrate with the film often leads to complex, self-organizing spatial patterns. Most importantly, the structural order of the film influences the magnetic, transport, catalytic, and electronic properties of the films. The understanding of structure-property relations in metal surfaces and the control of pattern formation is thus a key technological issue in modern nanoscience. In most cases, the growth mode of a metal film on top of a metal substrate is driven by a subtle interplay of adhesion energy and strain energy, the latter deriving from the different lattice parameters, or even bulk structure, of the two metals.

In heteroepitaxial systems, a mismatch is naturally present between the lattice spacing of the adsorbate and that of the substrate. This mismatch often leads to nontrivial surface patterning, which is typically self-organizing as driven by strain. For example, for the case of compact (111) surfaces, in absence of any alloying mechanisms, interesting regular patterns have been observed for the cases of $\mathrm{Cu}, \mathrm{Ni}$, or $\mathrm{Co}$ deposited on the compact surface of $\mathrm{Ru},{ }^{1-3}$ and similarly those of $\mathrm{Ni}$ on $\mathrm{Rh}(111), \mathrm{Co}$ on $\mathrm{Pd}(111)$, and $\mathrm{Cu}$ on $\mathrm{Pd}(111) .{ }^{4-6} \mathrm{In}$ all these cases, the thin film has a smaller lattice parameter than the substrate, namely, it experiences some tensile strain. But, a similar behavior is also shown in the compressive case, e.g., for Ag overlayers deposited on $\mathrm{Cu}(111)$ (Refs. 7-13) or on $\mathrm{Ni}(111) .{ }^{14}$ Once a patterned metal surface is formed, this can in turn be exploited to control the growth of reactants, ${ }^{15}$ the nucleation, and growth of graphene on metal films, ${ }^{16-20}$ the self-organization of two-dimensional (2D) vacancy islands at a heterogeneous metal interface, ${ }^{21,22}$ just to mention a few examples.

From a modeling point of view, different methodologies ranging from first-principles calculations to continuum models can shed light on the different processes involved. The main constraint to predicting the overlayer structure, based on the knowledge of the substrate and of the strain and adhesion energy of the adsorbate material, is the presence of multiple length scales involved in the phenomena. Metal surface patterning can indeed be characterized by periodicities of several nanometers in length. Any atomistic approach, for example, dealing with the modeling of an adsorbate at 1-ML coverage, would have to handle a system of at least 10000 atoms to be able to predict, with no a priori knowledge of the low-energy superstructure, the correct patterning behavior. Classical potentials coupled to Monte Carlo and molecular dynamics (MD) simulations have been used to explore surfaces at their ground state and in metastable configurations ${ }^{23,24}$ and to study basic diffusion and relaxation processes, but often this has been done relying on some previous knowledge of the emerging surface pattern. ${ }^{8}$

More recently, metal surface patterning has been examined using the phase-field-crystal (PFC) model. ${ }^{25-27}$ In this method, the local free energy of the system is expressed as a functional of a field related to the local atomic density. The free energy depends on a number of phenomenological parameters, including the elastic moduli of the material to be mimicked. The free energy is relaxed in a dissipative way, leading to the identification of ground-state and metastable configurations. Here, the typical time scales associated with free-energy relaxation on a metal surface are orders of magnitude larger than those accessible by MD simulations. Nevertheless, use of PFC and MD methods is limited by the need to resolve spatial details on the $\AA$ scale and can not be used to explore the sections of metallic surface that can be routinely probed in modern experiments.

To overcome the spatial limitation of the standard PFC, we will use a powerful extension of the PFC methodology through the so-called amplitude expansion of the density field. ${ }^{28-32}$ This methodology allows the study of 2D films adsorbed on a rigid substrate with spatial dimensions of several hundred nanometers, yet it retains atomistic resolution. We recently adopted this approach ${ }^{33}$ to study the ground and metastable states of heteroepitaxial ultrathin films, where the film lattice 
constant was smaller than that of the substrate (tensile mismatch). Here, we extend our calculations to include the compressive case, where the lattice constant ratio is reversed. We make predictions for the ground-state configurations of systems with different mismatches and compare them to experimental results, finding excellent agreement in both the tensile and the compressive regimes. In addition to the prediction of the ground-state configurations, we examine metastable states and kinetic trapping effects by means of nonequilibrium simulations.

The paper is organized as follows. In Sec. II, we explain how the PFC model is extended to the present case of strained overlayers. In Sec. III, the main results of the work are presented. In more detail, Sec. III A discusses the limits of very low substrate-overlayer coupling (incommensurate phase) and very large coupling (commensurate phase). Section III B describes the equilibrium structures of the overlayers as predicted by the model, for both the compressive and tensile mismatch cases, at intermediate coupling strengths, while Sec. III C examines the effect of an asymmetry in fcc and hep ordering. In Secs. III D and III E, nonequilibrium simulations are presented to examine metastable states and the ordering dynamics. A comparison of the results with experiments is discussed in Sec. III F. Finally, a discussion of the results and the conclusions that can be drawn from them are given in Sec. IV.

\section{MODEL}

To model the ordering of an atomic layer, an amplitude expansion of the phase-field-crystal model in two dimensions is employed here. Modeling the system in two dimensions does not allow for out-of-plane strains which could play a role for thick films, but is a reasonable approximation for the ultrathin metallic films considered here. The PFC model can be written in dimensionless form as

$$
\frac{\partial n}{\partial t}=\nabla^{2} \frac{\delta F}{\delta n}
$$

where $\mathcal{F}$ is the free-energy functional which can be written as $^{34-36}$

$$
\begin{aligned}
\frac{\mathcal{F}}{k_{B} T W \bar{\rho}}= & \int d \vec{r}\left[\frac{\Delta B}{2} n^{2}+\frac{B^{x}}{2} n\left(1+\nabla^{2}\right)^{2} n-\frac{t}{3} n^{3}\right. \\
& \left.+\frac{v}{4} n^{4}+V n\right] .
\end{aligned}
$$

The quantity $\bar{\rho}$ is the average number density, and $n$ is related to the dimensionless atomic-number density difference, ${ }^{37,38}$ $k_{B}$ is Boltzmann's constant, $T$ is temperature, and $W$ is the volume. On the right-hand side of Eq. (2), $\Delta B$ is proportional to a temperature difference, $B^{x}$ is proportional to the elastic moduli, and the parameters $t$ and $v$ control the magnitude of the amplitude fluctuations. In principle, the field $n$ is related to the atomic-number density $\rho$ and its average $\bar{\rho}$ through the equation $n \equiv(\rho-\bar{\rho}) / \bar{\rho}$. A more detailed explanation of the physical meaning of the parameters $\Delta B, B^{x}, t$, and $v$ can be found in Refs. 37 and 38. For the numerics conducted in this work, the parameters were set to $\left(\Delta B, B^{x}, t, v\right)=$ $\left(0.02,0.98, \frac{1}{2}, \frac{1}{3}\right)$.

In order to adapt the standard PFC formalism to the study of metal overlayers, the free-energy expression is modified by the addition of a term coupling the overlayer to the underlying substrate, represented by a pinning potential $V(x, y)$. In our model, the (111) or (0001) surface of the substrate (an fcc or hcp metal, respectively) is approximated by a rigid pinning dimensionless potential $V=U / k_{B} T$. The potential $V$ will be set to

$$
V=V_{0}\left(\sum_{j=1}^{3}(1+i \gamma) e^{i \vec{G}_{j}^{s} \cdot \vec{r}}+\text { c.c. }\right) \text {, }
$$

where c.c. is the complex conjugate, and $\vec{G}_{1}^{s}=-q^{s}(\sqrt{3} \hat{x} / 2+$ $\hat{y} / 2), \vec{G}_{2}^{s}=q^{s} \hat{y}$, and $\vec{G}_{3}^{s}=q^{s}(\sqrt{3} \hat{x} / 2-\hat{y} / 2)$ are the three reciprocal lattice vectors needed to reconstruct the potential of the (111) surface of the substrate, where $q_{s}=2 \pi / a_{s}$, with $a_{s}$ the lattice constant of the substrate. If $V_{0}$ is positive and $\gamma=0$, the maxima of the potential are placed on a triangular lattice mimicking the presence of the substrate atoms. The minima (adsorption sites) are ordered on a honeycomb lattice, containing two triangular sublattices. On real compact surfaces, these sublattices correspond to the adsorption with fcc or hcp stacking. When $\gamma=0$, the fcc and hcp minima are degenerate. This is a reasonable approximation since for many metal-on-metal systems, the difference between the adsorption energy on fcc and hcp sites is negligible, of the order of a few meV per atom. Nevertheless, a difference between the adsorption energy of hcp and fcc sites can be easily introduced in the model by changing the value of $\gamma$ which gives a phase shift in the potential such that fcc (or hcp) sites become more or less favorable. The influence of $\gamma \neq 0$ is indeed examined in Sec. III C.

The atomic ordering of the overlayer is represented in the standard PFC model by the dimensionless field $n$, which is uniform in the liquid state and periodic in the solid phase, i.e., it mimics the symmetry of a given crystalline state. This periodic variation limits the applicability of the model, as the grid spacing must be less than the atomic spacing in order to resolve atom positions in real space. To overcome this difficulty, an amplitude expansion was developed by Goldenfeld et al. ${ }^{28-32}$ In this formulation, the density is expanded around the basis set as the substrate, i.e.,

$$
n=\sum \eta_{j} e^{i \vec{G}_{j}^{s} \cdot \vec{r}}+\text { c.c. }
$$

where $\eta_{j}$ is a complex amplitude that varies on length scales much larger than $2 \pi /\left|\vec{G}_{j}^{s}\right|$, the film is such that $\vec{G}_{j}^{s}=\alpha \vec{G}_{j}^{f}$ and the mismatch between the substrate (lattice constant $a^{s}$ ) and film $\left(a^{f}\right)$ is then $\varepsilon \equiv\left(a^{s}-a^{f}\right) / a^{s}=(1-\alpha)$.

This approach has been used to describe commensurateincommensurate transitions, ${ }^{34,39}$ sliding friction, ${ }^{40,41}$ and the ordering of ultrathin binary films. ${ }^{35,36}$ All lengths are scaled such that the fundamental length scale is $a_{f} / 2 \pi$ and the amplitude of the coupling potential $V_{0}$ is also written in dimensionless form, whose relevance to experimental systems will be discussed in the following. The resulting equation of motion for the amplitudes, as done by Goldenfeld et al. ${ }^{28,29}$ and others, ${ }^{30-32}$ is

$$
\begin{aligned}
\frac{\partial \eta_{j}}{\partial t} \approx & -\frac{\delta F_{\eta}}{\delta \eta_{j}^{*}} \\
= & -\left[\Delta B+B^{x} \mathcal{G}_{j}^{2}+3 v\left(A^{2}-\left|\eta_{j}\right|^{2}\right)\right] \eta_{j} \\
& +2 t \prod_{i \neq j} \eta_{i}^{*}-V_{0}(1+i \gamma),
\end{aligned}
$$


where $\mathcal{G}_{j}=\nabla^{2}+2 i \alpha \vec{G}_{j}^{f} \cdot \vec{\nabla}+1-\alpha^{2}, A^{2} \equiv 2 \sum\left|\eta_{j}\right|^{2}$, and $F_{\eta}$ is the dimensionless free-energy functional:

$$
\begin{aligned}
\mathcal{F}_{\eta}= & \int d \vec{r}\left[\frac{\Delta B}{2} A^{2}+\frac{3 v}{4} A^{4}-2 t\left(\prod_{j=1}^{3} \eta_{j}+\text { c.c. }\right)\right. \\
& +\sum_{j=1}^{3}\left(B^{x}\left|\mathcal{G}_{j} \eta_{j}\right|^{2}-\frac{3 v}{2}\left|\eta_{j}\right|^{4}\right) \\
& \left.+V_{0}\left(\sum_{j}(1+i \gamma) \eta_{j}+\text { c.c. }\right)\right] .
\end{aligned}
$$

\section{RESULTS}

In the following sections, the equilibrium and transient patterns predicted by Eqs. (5) and (6) are presented. As discussed in the Introduction, it is a good approximation in many systems that the fcc and hcp sites have equal energy and consequently the results are given mainly for $\gamma=0$. Only in $\mathrm{Sec}$. III C is the influence of a nonzero $\gamma$ discussed; in all other sections, $\gamma=0$.

\section{A. Commensurate and incommensurate phases}

In this section, the states that minimize the free-energy functional [i.e., Eq. (6)] are presented as a function of the coupling strength $V_{0}$ and misfit strain $\varepsilon$. When the coupling strength is very low (i.e., the ratio $V_{0} / \varepsilon^{2} \rightarrow 0$ ), the overlayer floats above the substrate in an incommensurate state. The energy of this state can be calculated by substituting $\eta_{j}=$ $\phi \exp \left(i \varepsilon \vec{Q}_{j}^{f} \cdot \vec{r}\right)$ into Eq. (6) and minimizing with respect to $\phi$. This gives, for $\gamma=0$,

$$
\mathcal{F}_{\eta}^{i}=3 \Delta B \phi_{i}^{2}-4 t \phi_{i}^{3}+\frac{45 v}{2} \phi_{i}^{4},
$$

where $\phi_{i}$ is the value of $\phi$ that minimizes $\mathcal{F}_{\eta}^{i}$ and is given by

$$
\phi_{i}=\frac{t+\sqrt{t^{2}-15 v \Delta B}}{15 v} .
$$

At high $V_{0} / \varepsilon^{2}$, the adsorbate occupies the commensurate lattice sites, namely, a triangular sublattice either on the fcc or the hcp adsorption sites. This state is represented by $\eta_{j}=\phi e^{i \theta}$, where $\theta= \pm 2 \pi / 3$ is a phase shift that represents a shift to one of the two equivalent sublattices. Substitution into $\mathcal{F}$ gives

$$
\begin{aligned}
\mathcal{F}_{\eta}^{c}= & -3 \phi_{c} V_{0}+3\left[\Delta B+4 B^{x} \varepsilon^{2}(1-\varepsilon / 2)^{2}\right] \phi_{c}^{2} \\
& -4 t \phi_{c}^{3}+\frac{45 v}{2} \phi_{c}^{4} .
\end{aligned}
$$

The value of $\phi_{c}$ is obtained by solving $d \mathcal{F}_{\eta}^{c} / d \phi_{c}=0$, which is a cubic equation that can be solved analytically although the expression is somewhat lengthy. For the parameters used in the work, it turns out $\phi_{c} \approx \phi_{i}$, and in this limit

$$
\mathcal{F}_{\eta}^{c}-\mathcal{F}_{\eta}^{i} \approx-3 V_{0} \phi+12 B^{x} \varepsilon^{2} \phi^{2}
$$

(where we have also assumed $|\varepsilon| \ll 1$ ). This simple approximation clearly illustrates the main difference between the commensurate and incommensurate states. The energy in the commensurate state is lowered due to the pinning potential (i.e., $-3 V_{0} \phi$ ), but increased due to the elastic energy contribution (i.e., $12 B^{x} \varepsilon \phi^{2}$ ). In the absence of other minimum energy configurations, the transition from incommensurate to commensurate states should occur roughly when $V_{0}^{*}=$ $4 B^{x} \phi \varepsilon^{2}$. Of course, this transition is never observed due to the presence of other lower-energy configurations.

When a completely incommensurate layer is superimposed on the substrate, a honeycomb Moire pattern emerges that has a periodicity that is related to the different length scales of the layer and substrate. This periodicity is given by

$$
L_{M}=2 \pi /\left(\left|\vec{G}_{j}^{f}\right|-\left|\vec{G}_{j}^{s}\right|\right)=2 \pi / \varepsilon .
$$

Converting back to actual lengths, $\tilde{L}_{M}=a_{f} / \varepsilon$. Naturally, this length will play a role in the patterns that emerge in-between completely commensurate and incommensurate states.

\section{B. Equilibrium structures at intermediate couplings}

In order to construct an equilibrium phase diagram of all the ground-state surface structures, we performed numerical calculations by varying the coupling with the substrate and the mismatch, both in the tensile $\left(a^{f}<a_{s}\right)$ and compressive $\left(a^{f}>a_{s}\right)$ regimes. Our simulations started either from random configurations in which the overlayer atoms (or density peaks in the phase-field picture) occupy random positions on the substrate plane, or from configurations obtained from nonequilibrium runs (see Sec. IIID). In order to locate the lowest free-energy states of our overlayers, the free energy was minimized by discretization of Eq. (5) using Euler's method for the time difference and the discrete Laplacian introduced by Oono and Puri. ${ }^{42}$ The time step $\Delta t$ was set to 0.5 and the grid size to 2.0 (corresponding to $a_{f} / \pi$ in real units). In order to get to stable low-energy structures, typical relaxation times range from tens of thousands up to one million time units.

The stripe and honeycomb phases. Figure 1 shows the phase diagram of the overlayer, in both the compressive (left) and tensile (right) regimes. For small mismatches, namely $\varepsilon^{2}<30$, the phase diagram is dominated by two phases that we call the stripe and honeycomb phases. For larger mismatches, a third phase, called the triangular phase, emerges at intermediate couplings.

Let us focus at first on the stripe and honeycomb phases. In the stripe superstructure, the surface atoms periodically shift from fec to hcp sites relieving strain at the domain walls separating the two states. In the honeycomb superstructure, the atoms alternate between fcc and hcp domains that form a honeycomb pattern. Sample configurations are shown in Fig. 2.

From the phase diagram shown in Fig. 1 it is clear that, at a fixed mismatch value, the value of $V_{0}$ determines the stabilization of different phases. For low coupling values, the honeycomb phase is stabilized. As $V_{0}$ increases, the stripe phase becomes more stable and eventually the overlayer adopts the substrate epitaxy in the commensurate phase. We define $V_{0}^{h s}$ as the coupling strength of the honeycomb to stripe transition, and $V_{0}^{s c}$ as that of the stripe to commensurate transition. Both are roughly quadratic in $\varepsilon$, as might be expected since the gain in elastic energy is proportional to $\varepsilon^{2}$ and the loss in adsorption energy is proportional to $V_{0}$. 


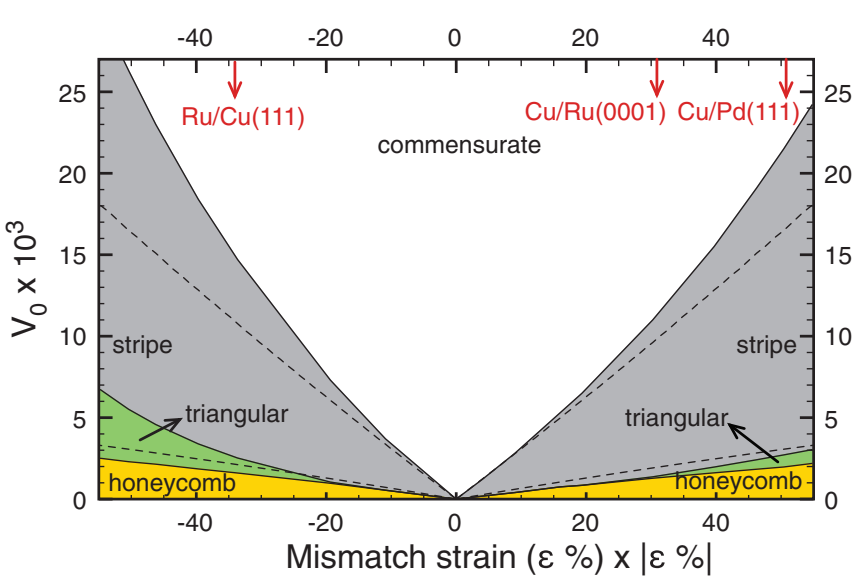

FIG. 1. (Color online) Phase diagram of the overlayer structures, as varying the coupling with the substrate $V_{0}$ ( $y$ axis) and the mismatch $\varepsilon$ ( $x$ axis). The compressive case corresponds to negative $\varepsilon$, on the left, while the tensile case to positive $\varepsilon$, on the right. The upper dashed line corresponds to the sine-Gordon prediction of the stripecommensurate transition and the lower dashed line corresponds to $V_{0}^{*}$, the potential at which the commensurate and floating incommensurate states have the same energy (see text for details).

The transition from a commensurate state to a stripe phase can be estimated by making a simplifying assumption that the phase of the $\eta_{j}$ 's act coherently and the amplitude of the $\eta_{j}$ 's is constant, i.e., assuming that $\eta_{j}=\phi e^{i \theta_{j}}$, where $\phi$ is constant
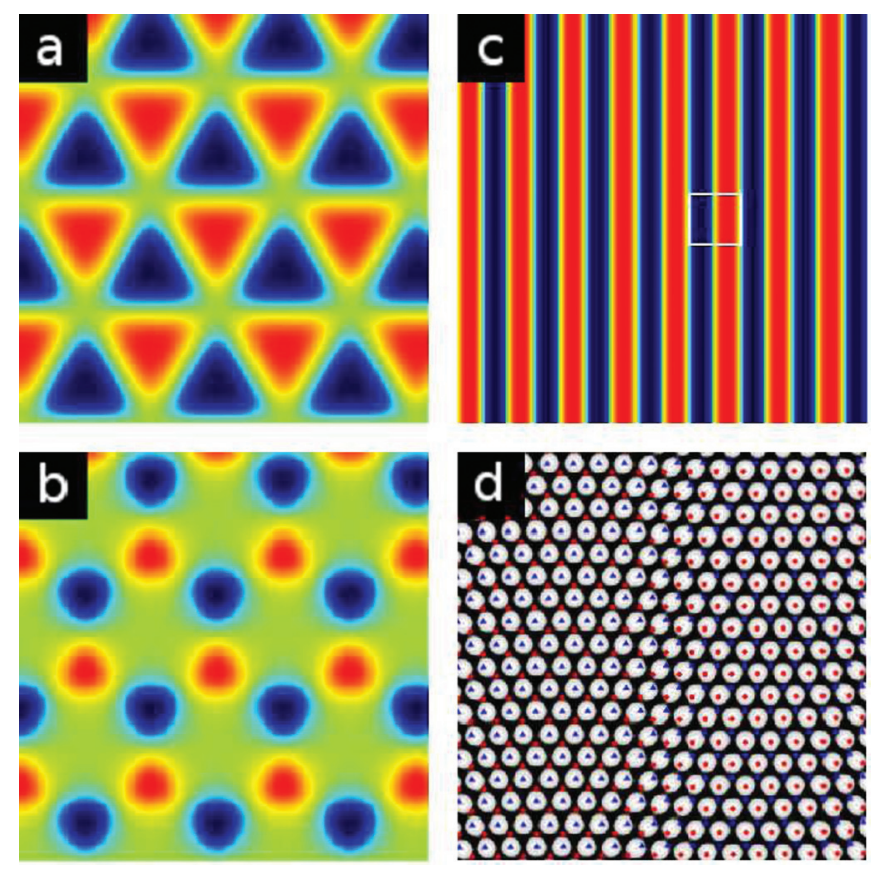

FIG. 2. (Color online) Snapshots of the equilibrium overlayer structures obtained for an overlayer on a (111) surface for a tensile strain of $\varepsilon=5.8 \%$. fcc domains are blue, hcp domains are red, and domain walls are green. Both (a) and (b) are honeycomb patterns at $V_{0}=0.00017$ and 0.0001 , respectively. Panel (c) is a stripe phase at $V_{0}=0.01$ and (d) is a reconstruction of the atomic density for the region enclosed by the white square in (c). The system sizes in (a), (b), (c), and (d) are $71 \times 71,61.6 \times 61.6,146.1 \times 146.1$, and $36.5 \times 36.5$ lattice constants, respectively.
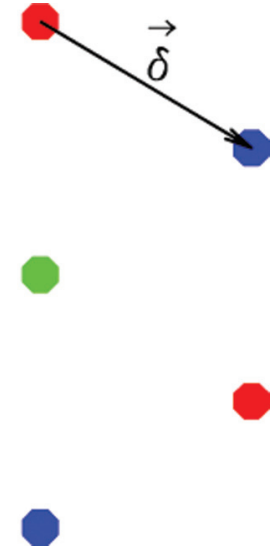

FIG. 3. (Color online) Schematic representation of the vector $\delta$ that links the fcc (blue) and hcp (red) sites.

and $\theta_{j} \approx \theta_{j}(-\infty)+\frac{2}{3} \vec{G}_{j} \cdot \vec{\delta} \Phi$, where $\vec{\delta}$ is a unit vector that points from an hep site to a fcc site as shown in Fig. 3.

Substituting these approximations into the free energy gives

$$
F_{\phi} \approx \int d \vec{r}\left[\frac{K}{2}\left(\frac{\partial \Phi}{\partial x}-\beta\right)^{2}-\frac{V_{0} \phi}{2} \cos (\Phi)\right],
$$

where $K \equiv 5 \alpha^{2} \phi^{2} B^{x} / 3, \beta \equiv 9\left(1-\alpha^{2}\right) /(5 \sqrt{3} \alpha)$, and the last term was obtained with the following approximation: $-\cos (\Phi / 3)-\sqrt{3} \sin (\Phi / 3)-\cos (2 \Phi / 3) / 2+\sqrt{3} \sin (2 \Phi / 3) /$ $2 \approx-5 / 4-\cos (\Phi) / 4$ for $0<\phi<2 \pi$. All terms not containing $\Phi$ have been dropped. While this form contains several approximations, it is conveniently written in a sine-Gordon form that can be solved exactly. The solution for the critical value of $V_{0}$ at the transition between commensurate and stripe states is then ${ }^{43}$

$$
V_{0}^{s c}=9 \pi^{2}\left(1-\alpha^{2}\right)^{2} B^{x} \phi / 20 \approx\left[9 \pi^{2} B^{x} \phi / 5\right] \varepsilon^{2},
$$

while the final approximation is valid for small $\varepsilon$. It should be noted [from Eq. (9)] that the value of $\phi$ that minimizes $F_{\eta}^{c}$ is a function of $V_{0}$ and thus Eq. (13) should be solved iteratively. As can be seen in Fig. 1, the prediction for $V_{0}^{s c}$ compares very well with the full solution in the small- $\varepsilon$ limit. It is not entirely unexpected that the sine-Gordon prediction breaks down at large $\varepsilon$ since the domain-wall width decreases as $\varepsilon$ increases and eventually the discrete nature of the lattice (still embedded in the amplitude expansion used here) begins to play a role. It is also likely that at high strains (and corresponding larger $V_{0}^{s c}$ values) that the approximation that $\phi$ is constant will break down. Unfortunately, the transition from the stripe phase to the ordered honeycomb superlattice is more difficult to predict due to the complex behavior of the amplitudes.

$V_{0}$ also affects the periodicity of the pattern within each phase region. In the top panel of Fig. 4, these periodicities are shown as a function of coupling strength at $\varepsilon=5.5 \%$ [appropriate for $\mathrm{Cu} / \mathrm{Ru}(0001)$, in the tensile regime] and $\varepsilon=-5.8 \%$ [appropriate for $\mathrm{Ru} / \mathrm{Cu}(111)$, in the compressive regime]. As expected, the periodicity of the honeycomb pattern starts at $L_{M}$ and then increases as $V_{0}$ increases in order to increase the size of the commensurate regions.

The periodicity of the stripe phase increases with $V_{0}$ and diverges at the transition to a commensurate state $\left(V_{0}^{s c}\right)$. This 

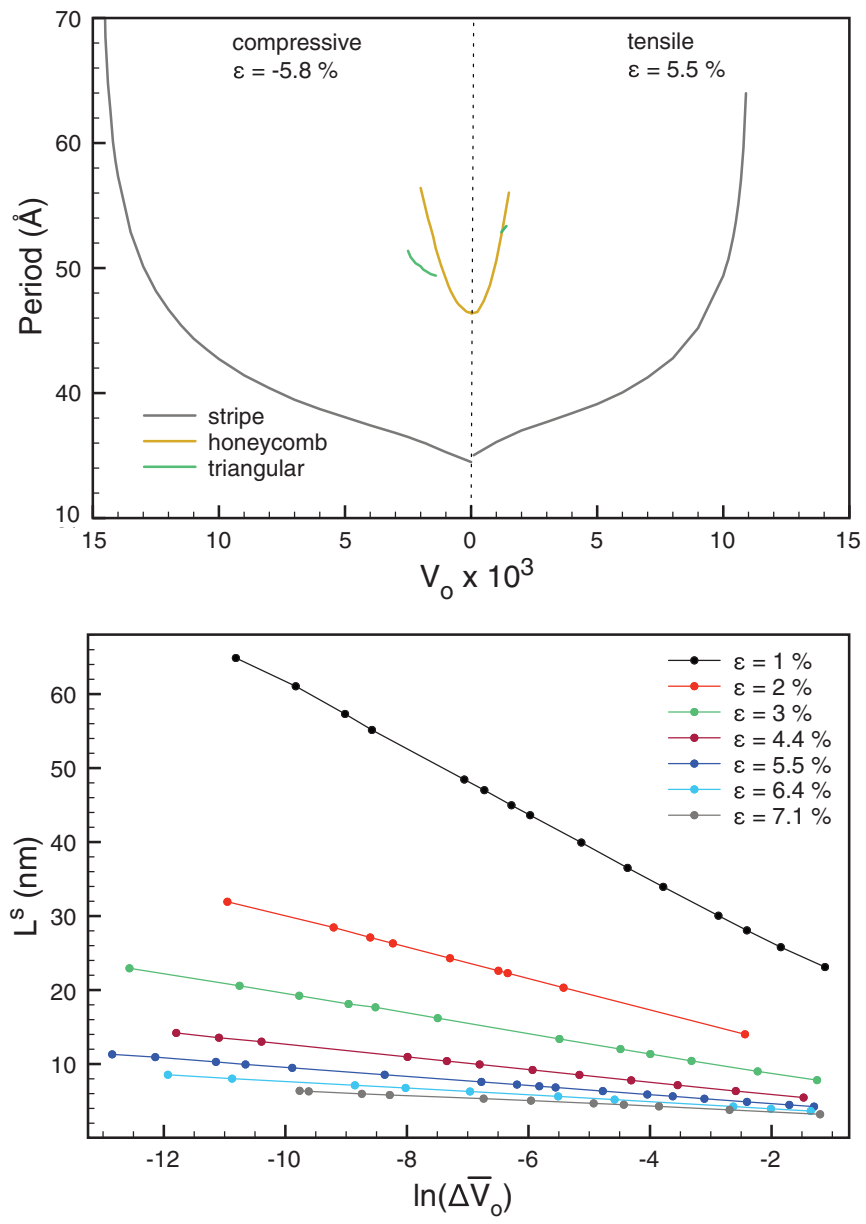

FIG. 4. (Color online) Top: Equilibrium wavelengths of the honeycomb (yellow), stripe (black), and triangular (blue) phases as a function of coupling strength. The data plotted on the graph refer to mismatch $\varepsilon=5.5 \%$ (tensile) and $\varepsilon=-5.8 \%$ (compressive). For these mismatch values, the triangular phase occupies a small portion of the phase diagram, in-between the honeycomb and stripe phases. At lower mismatch values, no triangular phase is expected. Bottom: The period of the stripe superstructure $\mathcal{L}^{s}$ is shown as a function of $V_{0}$ for various tensile strains.

transition is marked by a divergence in the stripe wavelength $\mathcal{L}^{s}$ as the transition is approached from below. More precisely, close to the transition,

$$
\mathcal{L}^{s} \approx \lambda \ln \left(\Delta \bar{V}_{0}\right)+C,
$$

where $\Delta \bar{V}_{0} \equiv\left(V_{0}^{c s}-V_{0}\right) / V_{0}^{s c}, \lambda$ is a characteristic length scale proportional to the domain-wall thickness, and $C$ is a constant. In this model, it can be shown that $\lambda \propto \sqrt{K /\left(V_{0}^{s c} \phi / 2\right)} \propto$ $\sqrt{\phi / V_{0}^{s c}}(1-\varepsilon)$. Confirmation of these predictions are shown in the bottom graph of Fig. 4.

The triangular phase. A third phase can be stabilized at large mismatches, and we call it the triangular phase. This phase appears as more relevant in the compressive regime (see Fig. 1), and was not included in our previous paper ${ }^{33}$ where we limited our analysis to the tensile regime. A snapshot of the triangular phase is shown in Fig. 5, as obtained from the minimization of an overlayer with a $-5.8 \%$ compressive mismatch $[\mathrm{Ru} / \mathrm{Cu}(111)]$ at the coupling potential $V_{0}=0.0024$.
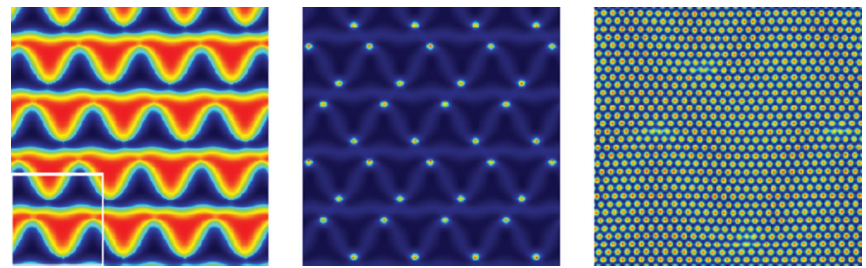

FIG. 5. (Color online) Snapshot of the triangular overlayer structure. Left: fcc domains are blue, hcp domains are red, and domain walls are green. Center: energy density plot. Brighter areas correspond to higher-energy regions. Dislocations appear as white spots. Right: atomic density reconstruction of the small white square region shown in the left panel. Four dislocations are visible in this image. The system size on the left and center panels is $92 \times 92$ lattice constants.

At variance with the stripe and honeycomb phases, the triangular phase is stabilized via the formation of a regular network of dislocations (see Fig. 5, center and right panels). This result is also consistent with observations of the "short period herringbone" structure observed in recent simulations of the full phase-field-crystal model with substrate coupling by Muralidharan et al. ${ }^{36}$

\section{Influence of finite $\gamma$}

The value of $\gamma$ determines which of the fcc or hcp ordering sites has the lower energy, such that for positive $\gamma$ the potential at the fcc sites is lower as shown in Fig. 6. In this plot, the values on the $y$ axis are chosen such that realistic fcc/hcp/bridge site differences for the $\gamma=0.03$ case, corresponding to the flat $\mathrm{Cu}(111)$ surface are obtained. Not surprisingly, it was found that increasing $\gamma$ tends to decreases the width of the hcp side of the stripe and increase the fcc side as shown in Fig. 7. Although the energy decreases as $\gamma$ increases, the transition to

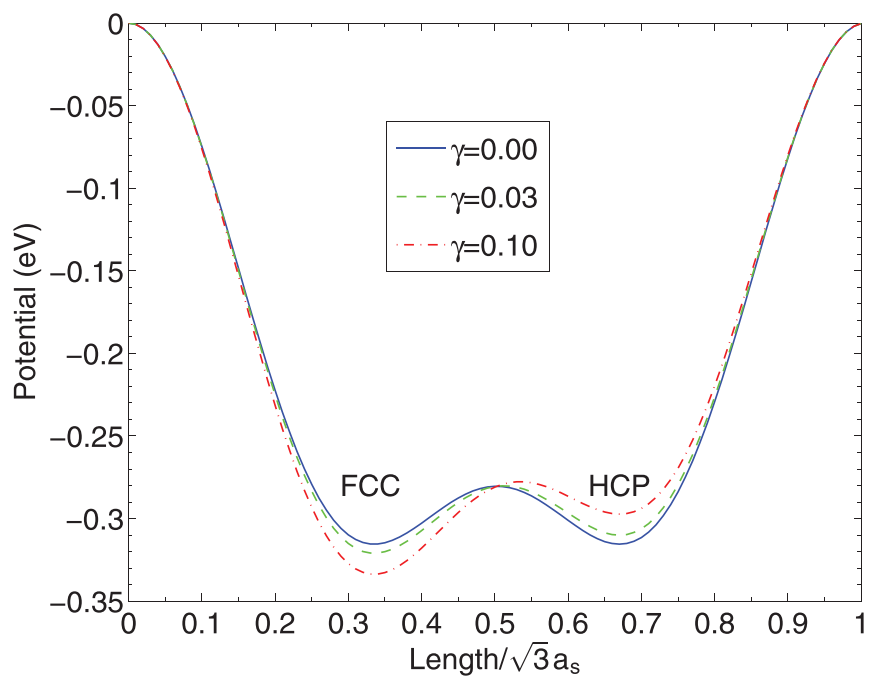

FIG. 6. (Color online) Potential profiles demonstrating the influence of $\gamma$. In this figure the profile of the pinning potential on a line that goes through both minima and two maxima for $\gamma=0.0$ (blue solid line), $\gamma=0.03$ (green dashed line), and $\gamma=0.10$ (red dashed-dotted line) are shown. The fcc site is the left minimum, hcp site is the right minimum, and bridge site is the local maximum between these minima. 


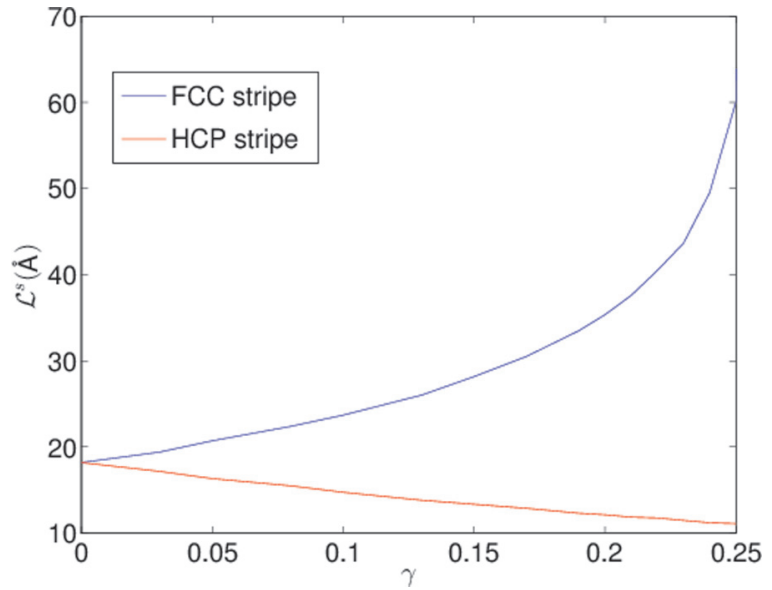

FIG. 7. (Color online) The influence of $\gamma$ on the stripe phase. The figure shows the equilibrium stripe wavelength $\mathcal{L}^{s}$ as a function of $\gamma$ for a case with $\alpha=1.058$ and $V_{0}=0.003$, separately for stripes having fcc (blue line) and hcp (red line) lattice ordering. The fcc stripes grow and diverge at some critical value of $\gamma=\gamma^{s c}$ while the hcp stripes shrink but remain finite with increasing $\gamma$.

the commensurate phase occurs at lower values of $V_{0}$, as the free energy of the commensurate phase decreases even faster. Thus, for each sufficiently large value of $\varepsilon$ and $V_{0}$, there is a critical value $\gamma=\gamma^{s c}$, where the wavelength of fcc stripes diverges and transition to the commensurate phase occurs.

\section{Nonequilibrium patterns}

Experimentally, atoms are deposited on the film over a finite time period to obtain the appropriate coverage and then annealed at a given temperature for some time. As discussed in the next section, decreasing the parameter $V_{0}$ is similar to increasing the number of layers. Thus, to mimic the deposition and annealing process, simulations were conducted starting at a relatively large $V_{0}$, i.e., in the commensurate region, and then $V_{0}$ was decreased linearly in time until a specified value was reached and then the system was relaxed for some time. Depending on the rate of variation of $V_{0}$, kinetic trapping is expected to play a role on the pattern formation. This is indeed the case, as shown in Fig. 8 for the case of compressive mismatch (the tensile mismatch case was discussed in our previous paper $^{33}$ ). The process started at $V_{0}=1.6 \times 10^{-3}$ from a random configuration that was relaxed at fixed $V_{0}$ for a few million steps until it contained large patches of fcc and hcp stacking, separated by domain walls that followed the symmetry directions of the substrate [Fig. 8(a) snapshot]. In this area of the phase diagram, $V_{0}$ was then reduced at the rate of $2 \times 10^{-9}$ per unit time. The stripe phase started to form, but due to kinetic trapping the system did not manage to achieve an ordered stripe phase [Fig. 8(b) snapshot] until $V_{0}$ was near the lower limit of the stripe region of the phase diagram. As the phase diagram in this region is more sensitive to the variations of $V_{0}$, we decreased the rate of variation of $V_{0}$ to $1 \times 10^{-9}$ per unit time. Nevertheless, the triangular phase was bypassed by the stabilization of the stripe phase [Fig. 8(c) snapshot] and the system finally converged to the honeycomb, incommensurate phase [Figs. 8(d) and 8(e) snapshots].

To highlight the kinetic trapping effects, $V_{0}$ was decreased further until it reached zero and then increased at a rate of $1 \times 10^{-9}$ until it reached a value of $4.4 \times 10^{-3}$, i.e., the same value as in configuration $8(\mathrm{~b})$. The resulting configuration is shown in Fig. 8(f) and is completely different than that obtained by decreasing $V_{0}$ from a large value. While this situation would be difficult to mimic in experiments (i.e., it would require

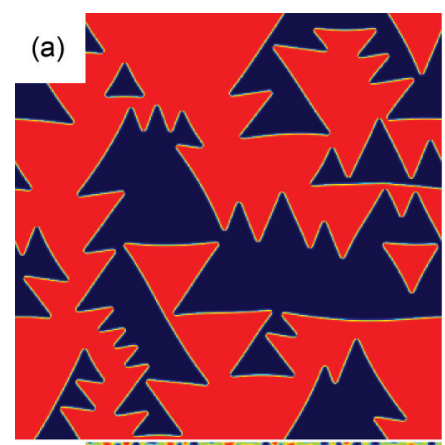

(d)

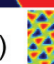

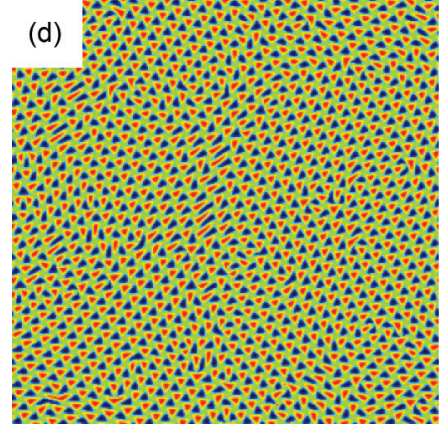
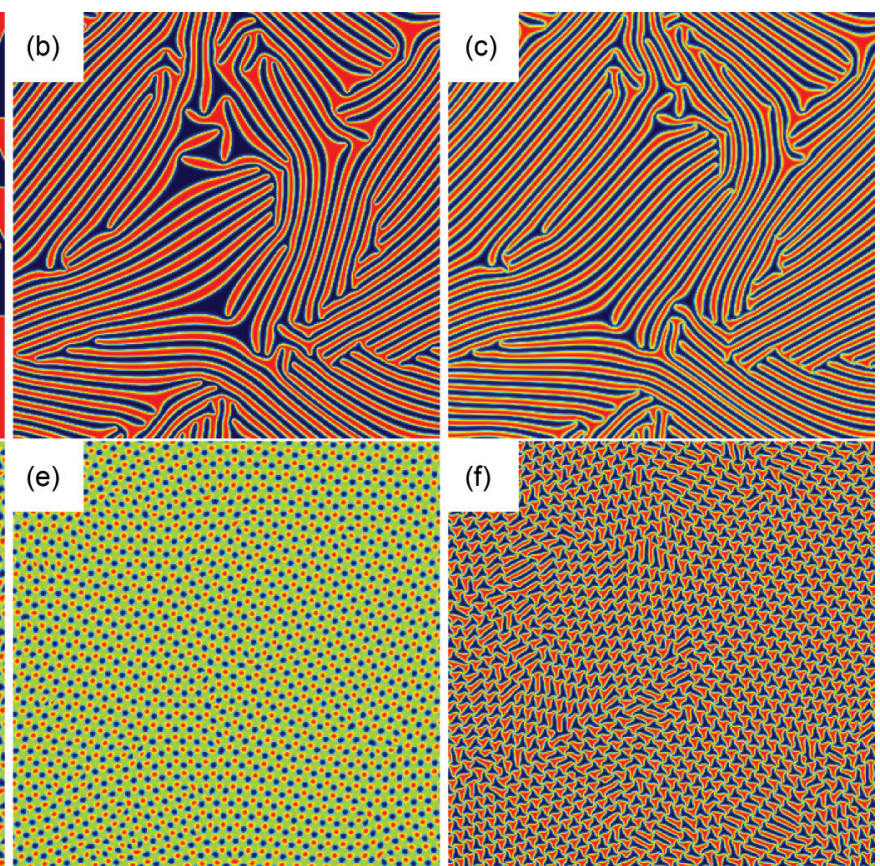

(e)

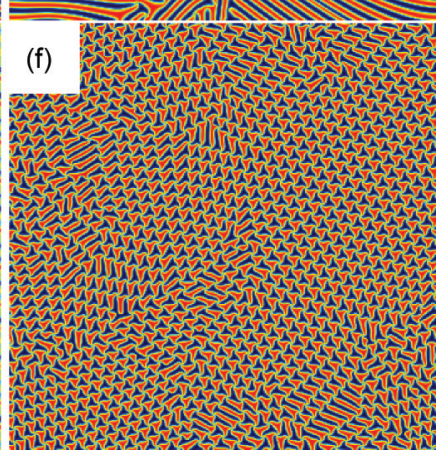

FIG. 8. (Color online) Snapshots from the nonequilibrium simulations in the compressive case, with $\varepsilon=-5.8 \%$ (see text for details). The snapshots (a)-(f) correspond to $V_{0}=15.6 \times 10^{-3}, 4.4 \times 10^{-3}, 2.0 \times 10^{-3}, 0.9 \times 10^{-3}, 0.5 \times 10^{-3}$, and $4.4 \times 10^{-3}$, respectively. Colors are as in Fig. 2. The size of each panel is $652 \times 652$ lattice constants, which corresponds to $0.166 \mu \mathrm{m} \times 0.166 \mu \mathrm{m} \mathrm{for} \mathrm{Ru} / \mathrm{Cu}(111)$. 


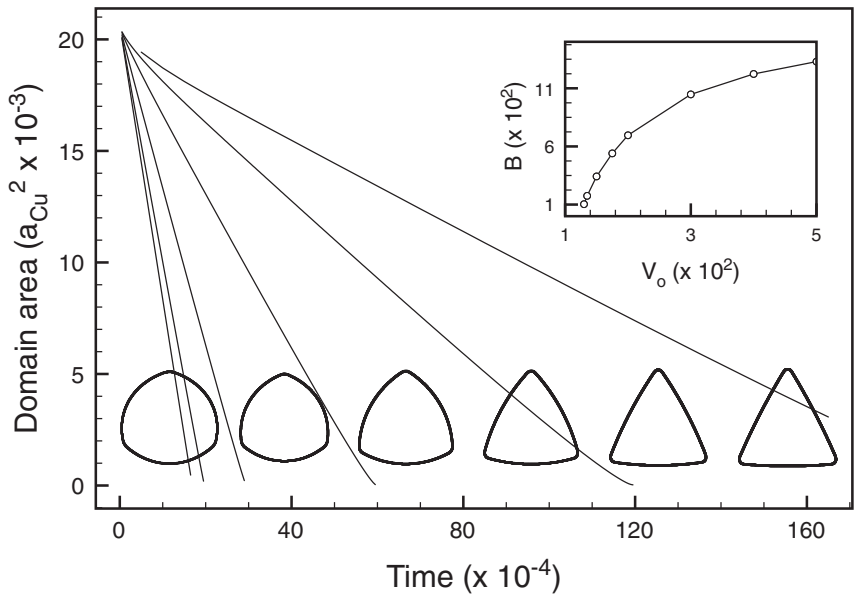

FIG. 9. Domain area as a function of time for $\varepsilon=5.5 \%$. In this figure, the domain area is plotted as a function of time for different values of $V_{0}$. For each value of $V_{0}$, the shape of the domain is illustrated when the area of the domain is $\approx 6000 a_{\mathrm{Cu}}^{2}$, where $a$ is the atomic spacing of the substrate. The slope $(A)$ of the curves in the linear regime is shown in the inset. From left to right, the lines and sample domains correspond to $V_{0}=$ $0.04,0.03,0.02,0.015,0.0135$, and 0.013 .

slowly removing atoms from the surface), it clearly highlights the history dependence of the film morphology.

\section{E. Domain growth dynamics}

In this section, we focus on the dynamics within the commensurate phase region in the $\gamma=0$ limit, where domains of hcp and fcc are energetically equivalent. In this region, the dynamics is driven to eliminate domain walls or interfaces that separate fcc and hcp regions. If we consider the case of a circular fcc domain, surrounded by a hcp phase, the curvature of the circular domain wall will lead the fcc domain to shrink and disappear. The average linear size of our circular domain will decrease as a square root of time, and its area will decrease linearly with time, ${ }^{44}$ i.e., $A / a_{\mathrm{Cu}}^{2}=A_{0} / a_{\mathrm{Cu}}^{2}-B t$, where $A_{0}$ is the initial area of the domain and $B$ is a function of $V_{0}$. Moreover, the domain pattern will be self-similar in time. ${ }^{44}$

In Figs. 9 and 10, the domain size is shown as a function of time for tensile and compressive films, respectively. As expected, the area of the domains decreases linearly in time. Even though the initial shape of the domain is circular, the domain quickly adopts a different shape, which depends on the coupling. This shape is then preserved during the whole dynamics. Just above $V_{0}^{s c}$, the domains are almost triangular with a dislocation at each vertex of the triangle, while far above $V_{0}^{s c}$ the domains are almost circular, although they still contain three partial dislocations.

In the inset of the figures, the slope $B$ is shown as a function of $V_{0}$. The rate of shrinkage of the domains depends on how close $V_{0}$ is to $V_{0}^{s c}$. It is interesting to note that $B$ goes to zero above and not at $V_{0}^{s c}$. The reason for this is that the dislocations at the corners of the domain get pinned by the potential. For example, for a tensile mismatch corresponding to $\mathrm{Cu} / \mathrm{Ru}(0001)$ (cf. Fig. 9), $V_{0}^{s c}=0.01113$, while the domain is pinned below $V_{0} \leqslant 0.0123$. We simulated the domain evolution in the commensurate phase also via the full phase-field crystal model.

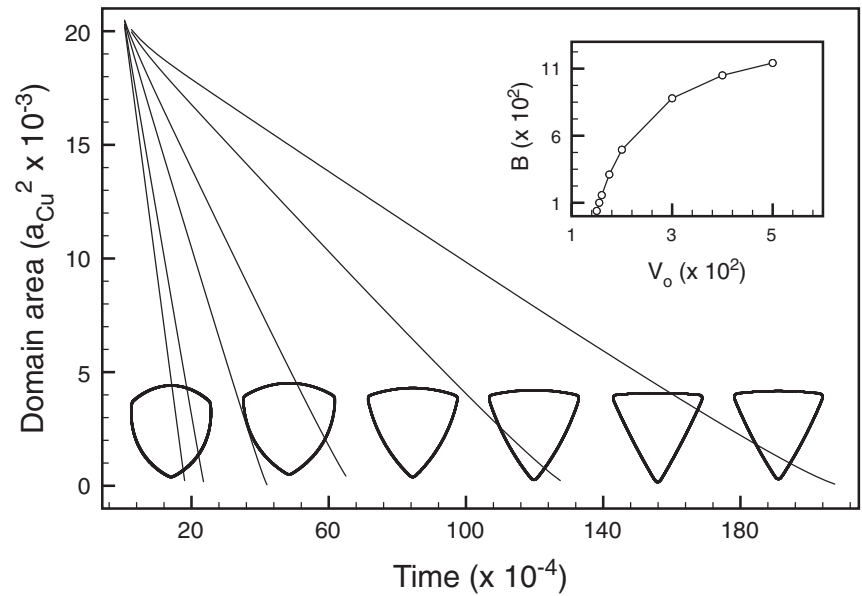

FIG. 10. Domain area as a function of time for $\varepsilon=-5.5 \%$. In this figure, the domain area is plotted as a function of time for different values of $V_{0}$. For each value of $V_{0}$, the shape of the domain is illustrated when the area of the domain is $\approx 6000 a_{\mathrm{Cu}}^{2}$, where $a$ is the atomic spacing of the substrate. The slope $(B)$ of the curves in the linear regime is shown in the inset. From left to right, the lines and sample domains correspond to $V_{0}=$ $0.05,0.03,0.02,0.0175,0.016$, and 0.0155 .

The results are consistent in all respects with those obtained via the amplitude expansion approach as shown in Fig. 11.

In the case of zero strain, slightly different behavior is observed. As seen in Fig. 12, the domain area decreases linearly with time for all values of $V_{0}$, however, the functional dependence of the slope $B$ on $V_{0}$ is different from the compressive and tensile cases. This is perhaps not surprising since at zero strain there is no crossover to a stripe phase. The exact domain shape and dependence of $B$ on $V_{0}$ are not easily

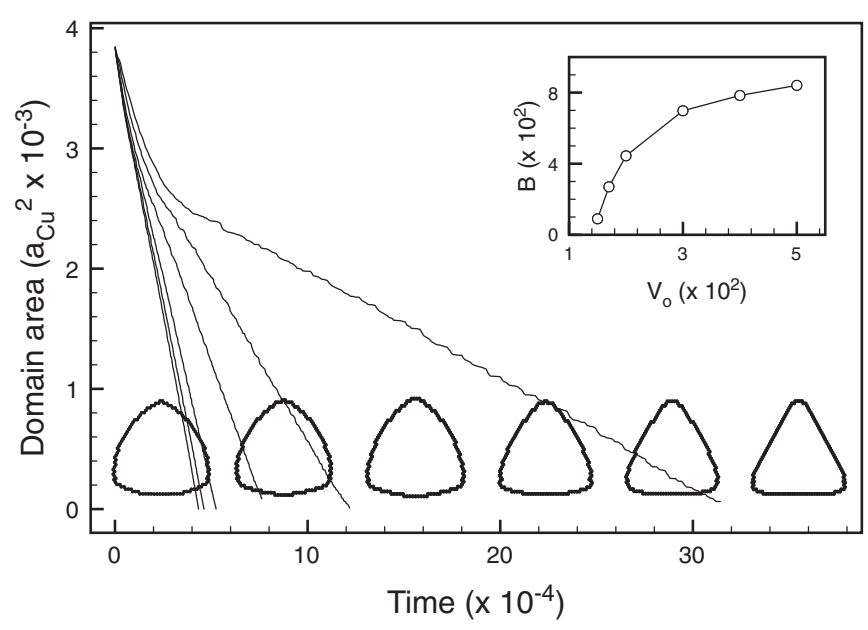

FIG. 11. Domain area as a function of time for $\varepsilon=5.5 \%$ for the phase-field-crystal model. In this figure, the domain area is plotted as a function of time for different values of $V_{0}$. For each value of $V_{0}$, the shape of the domain is illustrated when the area of the domain is $\approx 1360 a_{\mathrm{Cu}}^{2}$, where $a$ is the atomic spacing of the substrate. The slope $(B)$ of the curves in the linear regime is shown in the inset. From left to right, the lines and sample domains correspond to $V_{0}=$ $0.05,0.04,0.03,0.02,0.017$, and 0.015 . 


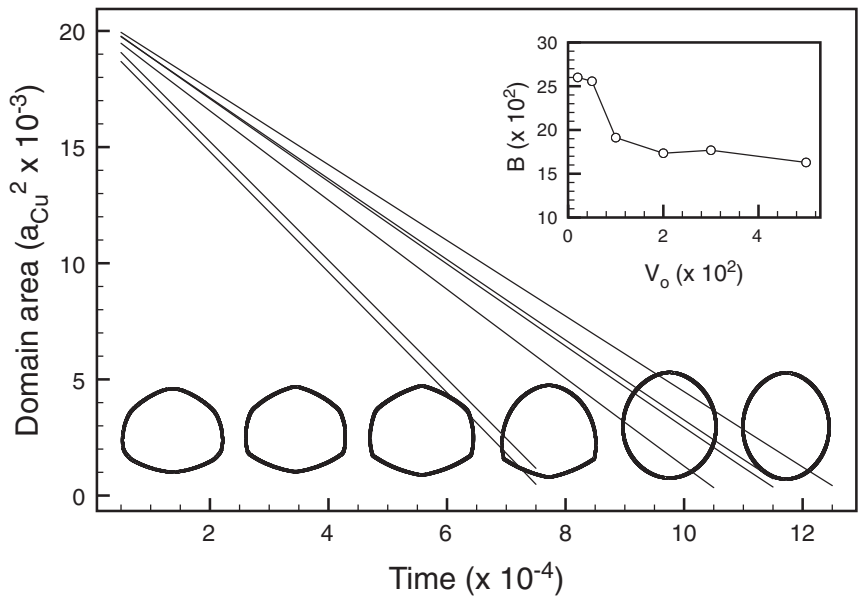

FIG. 12. Domain area as a function of time for $\varepsilon=0 \%$. In this figure, the domain area is plotted as a function of time for different values of $V_{0}$. For each value of $V_{0}$, the shape of the domain is illustrated when the area of the domain is $\approx 6000 a_{\mathrm{Cu}}^{2}$, where $a$ is the atomic spacing of the substrate. The slope $(A)$ of the curves in the linear regime is shown in the inset. From left to right, the lines and sample domains correspond to $V_{0}=0.05,0.03,0.01,0.005$, and 0.0002 .

explained. The only expectation is that the domain dynamics for very large $V_{0}$ should be the same for all strains.

As the domains shrink, the shape takes on a self-similar form, such that a domain at one time can be superimposed on an earlier or later drop if its size is appropriately rescaled. Examples of such scaling are shown in Fig. 13 for a strain of $\varepsilon=5.5 \%$, for three values of $V_{0}$. Similar scaling is also observed for the zero-strain and tensile cases.

\section{F. Comparison with experimental data and model predictions}

Experiments on $\mathrm{Cu} / \mathrm{Ru}(0001)$ (Refs. 2, 45, and 46) reveal patterns very similar to those predicted by the numerical simulations presented for the tensile case as discussed in a prior publication. ${ }^{33}$ More precisely, the stripe, triangular, and honeycomb structures seen in experiments at two, three, and four monolayers, respectively, were very similar to those obtained from simulations in which $V_{0}$ was lowered from 0.015 to $V_{0}=0.0032,0.00087$, and 0.00043 , respectively (see Ref. 33 for a direct comparison of simulation and experimental
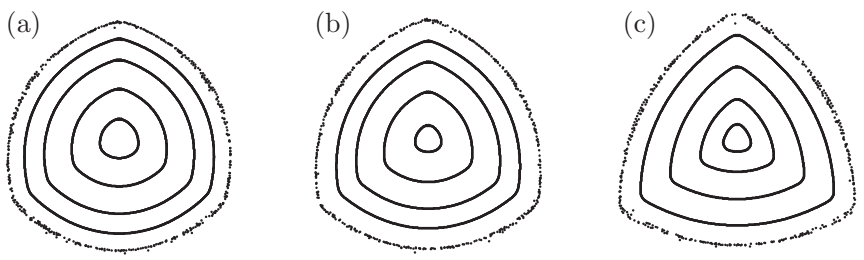

FIG. 13. Self-similar domains for various values of $V_{0}$. In (a), $V_{0}=0.04$ and the domains from outermost to innermost are at $t=$ $4 \times 10^{4}, 7.5 \times 10^{4}, 11 \times 10^{4}, 14.5 \times 10^{4}$, and $16.5 \times 10^{4}$. The inner domain shapes were also scaled to the size of the $t=4 \times 10^{4}$ domain and superimposed to highlight the self-similarity of the domain shape. Similar figures are presented in (b) for $V_{0}=0.03$ at times $t=6 \times 10^{4}$, $t=10.5 \times 10^{4}, t=14 \times 10^{4}, t=17.5 \times 10^{4}$, and $t=19.5 \times 10^{4}$ and in (c) for $V_{0}=0.02$ at times $t=7.5 \times 10^{4}, t=14.5 \times 10^{4}$, $t=22 \times 10^{4}, t=27 \times 10^{4}$, and $t=29 \times 10^{4}$. results). This comparison implies that increasing the number of layers is similar to decreasing the substrate film coupling constant $V_{0}$. To elucidate this comparison, it is useful to calculate how the ratio of the film-substrate interaction energy to elastic strain energy varies with the number of layers. Unfortunately, this is difficult to do for $\mathrm{Cu} / \mathrm{Ru}(0001)$ as the classical interaction potentials are not well characterized. However, it can be done for $\mathrm{Cu} / \mathrm{Pd}(111)$.

The $\mathrm{Cu} / \mathrm{Pd}(111)$ interface is characterized by a mismatch of $7.1 \%$. Experimental data ${ }^{47,48}$ point to layer-bylayer growth at room temperature. No alloying is observed and a recovery of the $\mathrm{Cu}$ incommensurate lattice spacing takes place for coverages larger than 4 ML. Unfortunately, no atomic resolution scanning tunneling microscope (STM) images of the surface are available. Our previous atomistic semiempirical calculations ${ }^{6}$ reveal the formation of hcp-fcc stacking domains already at very low coverages, in isolated $\mathrm{Cu}$ islands of monoatomic thickness, and for 1-ML coverage. In this respect, $\mathrm{Cu} / \mathrm{Pd}(111)$ differs from $\mathrm{Cu} / \mathrm{Ru}(0001)$, where the interaction with the substrate is strong enough to force $\mathrm{Cu}$ into the commensurate phase for 1-ML coverage. Our atomistic calculations were performed with the embedded atom potential parametrized by Foiles et al.$^{49} \mathrm{In}$ order to better compare the predictions of the semiempirical model to those of the 2D phase-field model, all energies in the following should be intended as energies per unit area. Our isolated $\mathrm{Cu}$ system consists of a single rectangular (111) layer of $450 \mathrm{Cu}$ atoms with lattice spacing $a$. Energies are calculated applying periodic boundary conditions along the in-plane directions. We define the strain energy $E_{\text {str }}$ as

$$
E_{\mathrm{str}}=E_{\mathrm{comm}}-E_{\mathrm{min}},
$$

where $E_{\mathrm{comm}}$ is the energy of the monolayer in the strained, Pd-commensurate phase $\left(a=a_{P d}\right)$, and $E_{\min }$ is the energy of the isolated $\mathrm{Cu}$ monolayer obtained by minimizing the energy with respect to $a\left(a=0.96 a_{\mathrm{Cu}}=0.96 \times 3.61=3.46 \AA\right)$.

In order to quantify the energy of interaction between the $\mathrm{Cu}$ monolayer and the $\mathrm{Pd}(111)$ surface, we built an fcc $\mathrm{Pd}$ slab $\left(a=a_{\mathrm{Pd}}=3.89 \AA\right)$ made by five (111) layers of 450 atoms each. A single $\mathrm{Cu}$ monolayer, in the Pd-commensurate state, was placed on top of the Pd slab as shown in Fig. 14 and the energy of the whole system was minimized. During the minimization, the three bottom Pd layers were kept fixed, while the two upper $\mathrm{Pd}$ layers and the $\mathrm{Cu}$ overlayer were free to relax along the $z$ direction. We define the $\mathrm{Cu} / \mathrm{Pd}(111)$ interaction energy $E_{\text {int }}$ as

$$
E_{\text {int }}=E_{\mathrm{tot}}-E_{\mathrm{sub}}-E_{\mathrm{min}},
$$

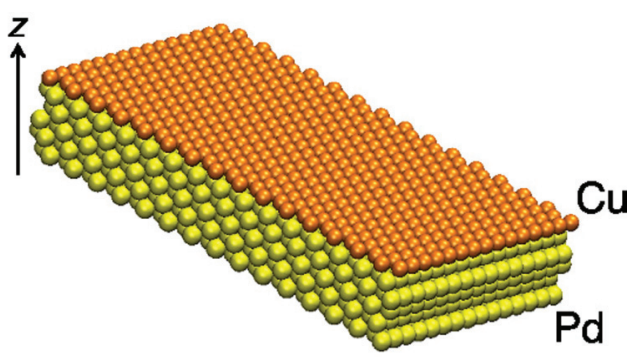

FIG. 14. (Color online) The $\mathrm{Cu} / \mathrm{Pd}(111)$ setup used for semiempirical calculations. 


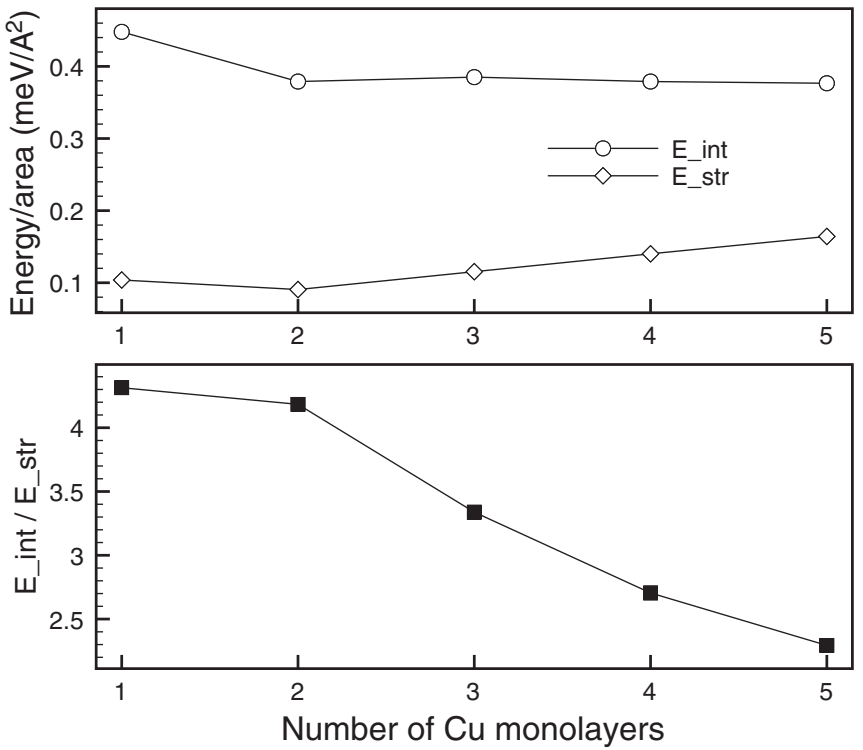

FIG. $15 . E_{\text {int }} / E_{\text {str }}, E_{\text {int }}$, and $E_{\text {str }}$ as calculated by the embedded atom method potential for the $\mathrm{Cu} / \mathrm{Pd}(111)$ case.

where $E_{\text {tot }}$ is the total minimized energy of the $\mathrm{Cu} / \mathrm{Pd}(111)$ system and $E_{\text {sub }}$ is the minimized energy of the isolated Pd slab. Our calculations provide $E_{\text {int }} / E_{\text {str }}=4.31$, for a single monolayer, which would correspond to $V_{0}=4.31 V_{0}^{*}=15 \times 10^{-3}$. From Fig. 1 this corresponds to a stripe superstructure, which is consistent with experiments and earlier MD studies. ${ }^{6}$

This procedure can be extended to the multilayer case. When increasing the number of $\mathrm{Cu}$ layers on top of the Pd slab, we do expect the interaction energy (per area) to decrease and finally converge when the $\mathrm{Cu}$ uppermost layers do not interact with the substrate any more. On the other hand, the strain energy (per area) of the $\mathrm{Pd}$-commensurate $\mathrm{Cu}$ film is going to increase as the number of layers increases. In Fig. 15, the $E_{\text {int }} / E_{\text {str }}$ ratio versus film thickness is plotted for the $\mathrm{Cu} / \mathrm{Pd}(111)$ case. The decrease of the $E_{\text {int }} / E_{\text {str }}$ guides us along the constant-mismatch vertical paths of the phase diagram shown in Fig. 1, leading the film to the incommensurate phase. These calculations are consistent with the idea that decreasing $V_{0}$ is similar to increasing the number of layers. Of course, it should be noted that the three-dimensional nature of multilayers leads to more than simply a change in interaction and elastic energy. More specifically, a changing in the stacking can lead to Shockley partial dislocations ${ }^{46}$ that can not be accounted for with our two-dimensional model.

For the compressive case, there are experiments on $\mathrm{Ag} / \mathrm{Cu}(111)$ (Refs. 7-13) and $\mathrm{Au} / \mathrm{Ni}(111)$ (Ref. 14) which reveal triangular patterns somewhat similar to the triangular patterns observed in the numerical simulations.

\section{CONCLUSIONS}

In this work, we have studied the formation of the complex patterns that form when a ultrathin films interact with a substrate that prefers a different lattice constant. The competition between the elastic strain induced by the substrate and the film-substrate interaction energy produces many different equilibrium structures as well as a host of metastable patterns. Our work predicts the structure of stable and metastable film configurations on length scales of several hundred nanometers, with atomic resolution, and allows for direct comparison with experiments. The predictions are in good agreement with existing experiments on $\mathrm{Cu} / \mathrm{Ru}(0001)$ and $\mathrm{Cu} / \mathrm{Pd}(111)$. Tests of our predictions would be significantly enhanced if new experiments were conducted on $\mathrm{Cu} / \mathrm{Pd}(111)$ that would image the patterns as a function of layer number. In contrast, tests of our predictions for $\mathrm{Cu} / \mathrm{Ru}(0001)$ would be enhanced if better theoretical or experimental information about the adhesion-elastic energy ratio were available.

It would also be quite interesting to examine the dynamics of ordering in many of the different phases. In this work, droplet ordering dynamics was examined in detail in the commensurate regions. Such an analysis would be interesting to do in other regions. For example, one could examine the shrinking (or growth) of a droplet of the stripe phase within the honeycomb structure or vice versa. Experimental realization of such dynamics could be achieved by local removal or addition of layers as done in recent experiments by Man et al. ${ }^{50}$

The use of complex amplitudes to model the surface ordering has several advantages that could be exploited in the future. For example, the model could be easily adapted to different substrates by simply changing the interaction potential and expanding the amplitudes around the basis of the substrate potential. In addition, since the amplitudes are relatively smooth, the use of adaptive mesh refinement schemes could be used to extend the work to even larger systems. It would also be interesting to extend this approach to true three-dimensional films. This would allow for partial dislocations that arise from different stackings and out-of-plane strains for thicker films. It would be quite interesting to observe how surface ordering interacts with surface instabilities in the latter case.

\section{ACKNOWLEDGMENTS}

K.R.E. acknowledges support from NSF under Grant No. DMR-0906676. This work has been supported in part by the Academy of Finland through its COMP CoE grant (Project No. 251748) and Aalto University School of Science Visiting Professor grant. E.G. was supported by Fundação de Amparo à Pesquisa do Estado de São Paulo - FAPESP (Grant No. 07/08492-9). We also thank CSC-IT Center for Science Ltd. for allocation of computational resources.
${ }^{1}$ F. E. Gabaly, W. L. W. Ling, K. F. McCarty, and J. de la Figuera, Science 308, 1303 (2005).

${ }^{2}$ C. Gunther, J. Vrijmoeth, R. Q. Hwang, and R. J. Behm, Phys. Rev. Lett. 74, 754 (1995).
${ }^{3}$ S.-F. Ding, S.-R. Deng, H.-S. Lu, Y.-L. Jiang, G.-P. Ru, D. W. Zhang, and X.-P. Qu, J. Appl. Phys. 107, 103534 (2010).

${ }^{4}$ A. Wander, C. J. Barnes, L. D. Mapledoram, and D. A. King, Surf. Sci. 281, 42 (1993). 
${ }^{5}$ M. Wasniowska, W. Wulfhekel, M. Przybylski, and J. Kirschner, Phys. Rev. B 78, 035405 (2008).

${ }^{6}$ J. Jalkanen, G. Rossi, O. Trushin, E. Granato, T. Ala-Nissila, and S.-C. Ying, Phys. Rev. B 81, 041412 (2010).

${ }^{7}$ W. E. McMahon, E. S. Hirschhorn, and T.-C. Chiang, Surf. Sci. Lett. 279, L231 (1992).

${ }^{8}$ I. Meunier, G. Treglia, J. M. Gay, B. Aufray, and B. Legrand, Phys. Rev. B 59, 10910 (1999).

${ }^{9}$ K. Umezawa, S. Nakanishi, M. Yoshimura, K. Ojima, K. Ueda, and W. M. Gibson, Phys. Rev. B 63, 035402 (2000).

${ }^{10}$ A. Bendounan, H. Cercellier, Y. Fagot-Revurat, B. Kierren, V. Y. Yurov, and D. Malterre, Appl. Surf. Sci. 212, 33 (2003).

${ }^{11}$ A. Bendounan, H. Cercellier, B. Kierren, Y. Fagot-Revurat, V. Y. Yurov, and D. Malterre, Europhys. Lett. 64, 392 (2003).

${ }^{12}$ F. Schiller, J. Cordón, D. Vyalikh, A. Rubio, and J. E. Ortega, Phys. Rev. Lett. 94, 016103 (2005).

${ }^{13}$ D. Malterre, B. Kierren, Y. Fagot-Revurat, C. Didiot, F. J. García de Abajo, F. Schiller, J. Cordón, and J. E. Ortega, New J. Phys. 13, 013026 (2011).

${ }^{14}$ J. Jacobsen, L. P. Nielsen, F. Besenbacher, I. Stensgaard, E. Laegsgaard, T. Rasmussen, K. W. Jacobsen, and J. K. Norskov, Phys. Rev. Lett. 75, 489 (1995).

${ }^{15}$ R. Otero, F. Calleja, V. M. Garcïa-Suärez, J. J. Hinarejos, J. de la Figuera, J. Ferrer, A. L. V. de Parga, and R. Miranda, Surf. Sci. 550, 65 (2004).

${ }^{16}$ P. Sutter, J. T. Sadowski, and E. Sutter, Phys. Rev. B 80, 245411 (2009).

${ }^{17}$ X. Li, W. Cai, J. An, S. Kim, J. Nah, D. Yang, R. Piner, A. Velamakanni, I. Jung, E. Tutuc et al., Science 324, 1312 (2009).

${ }^{18}$ R. Grantab, V. B. Shenoy, and R. S. Ruoff, Science 330, 946 (2010).

${ }^{19}$ J. F. Gao, J. Yip, J. J. Zhao, B. I. Yakobson, and F. Ding, J. Am. Chem. Soc. 133, 5009 (2011).

${ }^{20}$ Z. Sun, S. K. Hämäläinen, J. Sainio, J. Lahtinen, D. Vanmaekelbergh, and P. Liljeroth, Phys. Rev. B 83, 081415(R) (2011).

${ }^{21}$ K. Pohl, M. C. Bartelt, J. de la Figuera, N. C. Bartelt, J. Hrbek, and R. Q. Hwang, Nature (London) 397, 238 (1999).

${ }^{22}$ K. Ait-Mansour, A. Buchsbaum, P. Ruffieux, M. Schmid, P. Groning, P. Varga, R. Fasel, and O. Groning, Nano Lett. 8, 2035 (2008).

${ }^{23}$ T. Marten, O. Hellman, A. V. Ruban, W. Olovsson, C. Kramer, J. P. Godowski, L. Bech, Z. Li, J. Onsgaard, and I. A. Abrikosov, Phys. Rev. B 77, 125406 (2008).

${ }^{24}$ A. Bergbreiter, H. E. Hoster, S. Sakong, A. Grob, and R. J. Behm, Phys. Chem. Chem. Phys. 9, 5127 (2007).

${ }^{25}$ K. R. Elder, M. Katakowski, M. Haataja, and M. Grant, Phys. Rev. Lett. 88, 245701 (2002).

${ }^{26}$ K. R. Elder and M. Grant, Phys. Rev. E 70, 051605 (2004).
${ }^{27}$ K. R. Elder, N. Provatas, J. Berry, P. Stefanovic, and M. Grant, Phys. Rev. B 75, 064107 (2007).

${ }^{28}$ N. Goldenfeld, B. P. Athreya, and J. A. Dantzig, Phys. Rev. E 72, 020601(R) (2005).

${ }^{29}$ B. P. Athreya, N. Goldenfeld, and J. A. Dantzig, Phys. Rev. E 74, 011601 (2006)

${ }^{30}$ D. H. Yeon, Z.-F. Huang, K. R. Elder, and K. Thornton, Philos. Mag. 90, 237 (2010).

${ }^{31}$ K. R. Elder, Z.-F. Huang, and N. Provatas, Phys. Rev. E 81, 011602 (2010).

${ }^{32}$ Z.-F. Huang, K. R. Elder, and N. Provatas, Phys. Rev. E 82, 021605 (2010).

${ }^{33}$ K. R. Elder, G. Rossi, P. Kanerva, F. Sanches, S.-C. Ying, E. Granato, C. V. Achim, and T. Ala-Nissila, Phys. Rev. Lett. 108, 226102 (2012)

${ }^{34}$ C. V. Achim, M. Karttunen, K. R. Elder, E. Granato, T. Ala-Nissila, and S. C. Ying, Phys. Rev. E 74, 021104 (2006).

${ }^{35}$ S. Muralidharan and M. Haataja, Phys. Rev. Lett. 105, 126101 (2010).

${ }^{36}$ S. Muralidharan, R. Khodadad, E. Sullivan, and M. Haataja, Phys. Rev. B 85, 245428 (2012).

${ }^{37}$ A. Jaatinen and T. Ala-Nissila, Phys. Rev. E 82, 061602 (2010).

${ }^{38}$ A. Jaatinen, C. V. Achim, K. R. Elder, and T. Ala-Nissila, Phys. Rev. E 80, 031602 (2009).

${ }^{39}$ J. A. P. Ramos, E. Granato, C. V. Achim, S. C. Ying, K. R. Elder, and T. Ala-Nissila, Phys. Rev. E 78, 031109 (2008).

${ }^{40}$ C. V. Achim, J. A. P. Ramos, M. Karttunen, K. R. Elder, E. Granato, T. Ala-Nissila, and S. C. Ying, Phys. Rev. E 79, 011606 (2009).

${ }^{41}$ J. A. P. Ramos, E. Granato, S. C. Ying, C. V. Achim, K. R. Elder, and T. Ala-Nissila, Phys. Rev. E 81, 011121 (2010).

${ }^{42}$ Y. Oono and S. Puri, Phys. Rev. A 38, 434 (1988).

${ }^{43}$ P. M. Chaikin and T. C. Lubensky, Principles of Condensed Matter Physics (Cambridge University Press, Cambridge, UK, 1995).

${ }^{44}$ H. E. Stanley, Introduction to Phase Transitions and Critical Phenomena (Oxford University Press, New York, 1971).

${ }^{45}$ A. K. Schmid, N. C. Bartelt, J. C. Hamilton, C. B. Carter, and R. Q. Hwang, Phys. Rev. Lett. 78, 3507 (1997).

${ }^{46}$ J. de la Figuera, A. K. Schmid, N. C. Bartelt, K. Pohl, and R. Q. Hwang, Phys. Rev. B 63, 165431 (2001).

${ }^{47}$ G. Liu, T. P. S. Clair, and D. W. Goodman, J. Phys. Chem. B 103, 8578 (1999).

${ }^{48}$ A. de Siervo, E. A. Soares, R. Landers, and G. G. Kleiman, Surf. Sci. 575, 115417 (2005).

${ }^{49}$ S. M. Foiles, M. I. Baskes, and M. S. Daw, Phys. Rev. B 33, 7983 (1986).

${ }^{50}$ K. L. Man, M. C. Tringides, M. M. T. Loy, and M. S. Altman, Phys. Rev. Lett. 101, 226102 (2008). 